\title{
Infinitely many solutions for a class of p-Laplacian equation with Sturm-Liouville type nonhomogeneous boundary conditions
}

\author{
Fenglong Sun ${ }^{\mathrm{a}}$, Lishan Liu ${ }^{\mathrm{a}, \mathrm{b}, *}$, Yonghong Wu ${ }^{\mathrm{b}}$ \\ a School of Mathematical Sciences, Qufu Normal University, Qufu 273165, Shandong, People's Republic of China. \\ ${ }^{b}$ Department of Mathematics and Statistics, Curtin University, Perth, WA6845, Australia.
}

Communicated by X. Z. Liu

\begin{abstract}
We establish the criteria for the existence of infinitely many solutions for a class of one-dimensional p-Laplacian equations with Sturm-Liouville type nonhomogeneous boundary conditions. The nonlinear term has two parameters $\lambda, \mu$ and is dependent on $x$ and the derivative $u^{\prime}(x)$ of the solution to be determined. The main method used for the study is Ricceri's Variational Principle. (C) 2017 All rights reserved.
\end{abstract}

Keywords: Infinitely many solutions, p-Laplacian equation, nonhomogeneous boundary conditions, variational method. 2010 MSC: 34B15, 47J10.

\section{Introduction}

In this paper, we are concerned with the existence of infinitely many solutions to the following SturmLiouville type nonhomogeneous boundary value problem

$$
\left\{\begin{aligned}
&-\left(\phi_{p}\left(u^{\prime}\right)\right)^{\prime}=( \lambda f(x, u(x))+\mu g(x, u(x)) \\
&\left.+\int_{0}^{u^{\prime}(x)} \frac{\partial}{\partial x}\left(\frac{(p-1)|\tau|^{p-2}}{h(x, \tau)}\right) d \tau\right) h\left(x, u^{\prime}(x)\right), \quad x \in(a, b), \\
& \alpha u(a)-\beta u^{\prime}(a)=A, \quad \gamma u(b)+\sigma u^{\prime}(b)=B
\end{aligned}\right.
$$

where $\lambda>0$ and $\mu \geqslant 0$ are parameters, $p>1, \phi_{p}(t)=|t|^{p-2} t, \alpha, \gamma, \beta, \sigma>0$, and $A$, B are arbitrary constants. The function $h:[a, b] \times \mathbb{R} \rightarrow \mathbb{R}$ satisfies the following conditions:

(i) $0<m:=\inf _{(x, t) \in[a, b] \times \mathbb{R}} h(x, t) \leqslant M:=\sup _{(x, t) \in[a, b] \times \mathbb{R}} h(x, t) ;$

(ii) the function $t \mapsto h(x, t)$ is continuous for all $x \in[a, b]$ and the function $x \mapsto h(x, t)$ is in $C^{1}([a, b])$ for all $t \in \mathbb{R}$.

\footnotetext{
*Corresponding author

Email addresses: sfenglong@sina.com (Fenglong Sun), mathlls@163.com (Lishan Liu), Y. Wu@curtin.edu . au (Yonghong Wu)
} doi:10.22436/jnsa.010.11.37 
It is easy to see the term $\int_{0}^{\mathfrak{u}^{\prime}(x)} \frac{\partial}{\partial x}\left(\frac{(p-1)|\tau|^{p-2}}{h(x, \tau)}\right) d \tau$ in equation (1.1) will vanish provided $h$ being independent of $x$. We also assume that the functions $f, g:[a, b] \times \mathbb{R} \rightarrow \mathbb{R}$ are $L^{1}$-Carathéodary functions. Here, $a$ function $f:[a, b] \times \mathbb{R} \rightarrow \mathbb{R}$ is said to be an $\mathrm{L}^{1}$-Carathéodary function, provided that

(a) the function $x \mapsto f(x, t)$ is measurable for every $t \in \mathbb{R}$;

(b) the function $t \mapsto f(x, t)$ is continuous for almost every $x \in[a, b]$;

(c) for every $\rho>0$ there exists a function $l_{\rho} \in \mathrm{L}^{1}([a, b])$ such that

$$
\sup _{|t| \leqslant \rho}|f(x, t)| \leqslant l_{\rho}(x)
$$

for almost every $x \in[a, b]$.

According to the critical point theory, the study of the existence of solutions for the problem (1.1) can be transformed into the study of the existence of critical points for some functional $\Phi-\lambda \Psi$ associated with the problem (1.1).

In [24], Ricceri established a famous variational principle for the existence of at least three critical points of the functional $\Phi-\lambda \Psi$ when the parameter $\lambda$ lies in some interval $\Lambda \subset \mathbb{R}$. In [23], Ricceri established a similar variational principle associated with infinitely many critical points. In the last decade or so, as a useful method to obtain the existence or multiplicity results, Ricceri's Variational Principle has been extended and used widely to study many problems including: Kirchhoff-type problems ([1, 12, 15, $19,20,26])$, problems with impulsive effect $([6,11,21,29])$, fractional differential equations ([27, 32]), p-Laplacian or $p(x)$-Laplacian equations $([2-4,13,14,16])$, Yamabe equations ([9]), superlinear discrete problems ([8]), non-differential functionals $([5,7,22])$, and many other problems (see $[17,25,30]$ and the references therein).

In [17], the authors obtained the existence of at least three classical solutions to the quasilinear elliptic system

$$
\left\{\begin{array}{l}
-\left(p_{i}-1\right)\left|u_{i}^{\prime}(x)\right|^{p_{i}-2} u_{i}^{\prime \prime}(x)=\left[\lambda F_{u_{i}}\left(x, u_{1}, \ldots, u_{n}\right)+\mu G_{u_{i}}\left(x, u_{1}, \ldots, u_{n}\right)\right] h_{i}\left(x, u_{i}^{\prime}(x)\right), \quad x \in(a, b), \\
u_{i}(a)=u_{i}(b)=0, \text { for } i=1,2, \ldots, n,
\end{array}\right.
$$

where $p_{i}>1(i=1, \ldots, n), \lambda>0$ and $\mu \geqslant 0$ are parameters, $h_{i}:[a, b] \times \mathbb{R} \rightarrow \mathbb{R}$ is a bounded continuous function such that $m_{i}:=\inf _{(x, t) \in[a, b] \times \mathbb{R}} h_{\mathfrak{i}}(x, t)>0, F:[a, b] \times \mathbb{R}^{n} \rightarrow \mathbb{R}$ is a function such that the mapping $\left(t_{1}, t_{2}, \ldots, t_{n}\right) \mapsto F\left(x, t_{1}, t_{2}, \ldots, t_{n}\right)$ is $C^{1}$ in $\mathbb{R}^{n}$ for all $x \in[a, b], F_{t_{i}}(i=1, \ldots, n)$ is continuous in $[a, b] \times \mathbb{R}^{n}$, and $F(x, 0, \ldots, 0)=0$ for all $x \in[a, b], G:[a, b] \times \mathbb{R}^{n} \rightarrow \mathbb{R}$ is a function such that the mapping $\left(t_{1}, t_{2}, \ldots, t_{n}\right) \mapsto G\left(x, t_{1}, t_{2}, \ldots, t_{n}\right)$ is $C^{1}$ in $\mathbb{R}^{n}$ for all $x \in[a, b]$ and $G_{t_{i}}(i=1, \ldots, n)$ is continuous in $[a, b] \times \mathbb{R}^{n}$. But they had a miscalculation in the proof of Lemma 2.2 in [17], since $h_{i}$ was also dependent on $x$.

In [28], the authors obtained the existence of at least three generalized solutions for the following second-order Sturm-Liouville boundary value problem:

$$
\left\{\begin{array}{l}
\left(\phi_{p}\left(u^{\prime}\right)\right)^{\prime}+\lambda f(t, u) h\left(u^{\prime}\right)=0, \quad x \in(a, b), \\
\alpha u(a)-\beta u^{\prime}(a)=0, \quad \gamma u(b)+\sigma u^{\prime}(b)=0,
\end{array}\right.
$$

where $\lambda>0$ is a parameter, $p>1, \alpha, \gamma \geqslant 0, \beta, \sigma>0, f:[a, b] \times \mathbb{R} \rightarrow \mathbb{R}$ is an $\mathrm{L}^{1}$-Carathéodary function, and $h: \mathbb{R} \rightarrow \mathbb{R}$ is a bounded continuous function such that $\inf _{t \in \mathbb{R}} h(t)>0$. In [18], the authors obtained the existence of infinitely many classical solutions to the following $p_{i}$-Laplacian systems with Sturm-Liouville boundary conditions

$$
\left\{\begin{array}{l}
-\left(\phi_{p_{i}}\left(u_{i}^{\prime}\right)\right)^{\prime}=\lambda F_{u_{i}}\left(x, u_{1}, \ldots, u_{n}\right) h_{i}\left(u_{i}^{\prime}(x)\right), \quad x \in(a, b), \\
\alpha_{i} u_{i}(a)-\beta_{i} u_{i}^{\prime}(a)=0, \quad \gamma_{i} u_{i}(b)+\sigma_{i} u_{i}^{\prime}(b)=0,
\end{array}\right.
$$

where $\lambda>0$ is a parameter, $\alpha_{i}, \gamma_{i} \geqslant 0, \beta_{i}, \sigma_{i}>0(i=1,2, \ldots, n)$. 
The purpose of the present paper is to extend the boundary value problems in $[17,18,28]$ to a more general case. In addition, to overcome the difficulty caused by the dependence of $h(x, t)$ on $x$, we add an extra term (i.e. $\int_{0}^{u^{\prime}(x)} \frac{\partial}{\partial x}\left(\frac{(p-1)|\tau|^{p-2}}{h(x, \tau)}\right) d \tau$ ) in the nonlinearity, then the form of the functional $\Phi$ in [17] can still be used here and the classical and weak solutions to problem (1.1) coincide, see (2.2) and Lemma 2.4 .

The present paper is built up as follows. In Section 2, we give some notations, the definitions of functionals, classical solutions, weak solutions, and the relationship between them. We also prove some estimates and regularity assumptions for the functionals $\Phi$ and $\Psi$. We recall the variational principle at the end of this section. In Section 3, we give the main results of the existence of infinitely many solutions in Theorem 3.1 and Theorem 3.5, with some corollaries. In Section 4, we give an example to illustrate the application of our results.

\section{Preliminaries and lemmas}

Let $X$ be the Sobolev space $W^{1, p}([a, b])$ endowed with the norm

$$
\|\mathrm{u}\|:=\left(\int_{a}^{b}|u(t)|^{p}+\left|u^{\prime}(t)\right|^{p} d t\right)^{\frac{1}{\mathfrak{p}}}, \quad \forall u \in X .
$$

It is easy to see that the space $(X,\|\cdot\|)$ is a real reflexive Banach space and $\max \left\{\|u\|_{L^{p}},\left\|u^{\prime}\right\|_{L^{p}}\right\} \leqslant\|u\|$ for each $u \in X$. By the Sobolev embedding theorem (see [10]), $X$ is compactly embedded into $C([a, b])$. We also denote $\|\cdot\|_{\infty}$ as the usual norm of $L^{\infty}([a, b])$.

Next, define the functions

and

$$
\mathrm{J}_{\chi}(\mathrm{s})=\mathrm{J}(\mathrm{x}, \mathrm{s}):=\int_{0}^{\mathrm{s}} \frac{(\mathrm{p}-1)|\delta|^{\mathrm{p}-2}}{\mathrm{~h}(\mathrm{x}, \delta)} \mathrm{d} \delta, \quad \forall x \in[\mathrm{a}, \mathrm{b}], \mathrm{s} \in \mathbb{R}
$$

$$
\mathrm{H}_{x}(\mathrm{~s})=\mathrm{H}(x, \mathrm{~s}):=\int_{0}^{s} \mathrm{~J}(x, \tau) \mathrm{d} \tau, \quad \forall x \in[\mathrm{a}, \mathrm{b}], \mathrm{s} \in \mathbb{R}
$$

For any fixed $x \in[a, b]$, the fact that $H_{\chi}^{\prime \prime}(s)=J_{\chi}^{\prime}(s)=\frac{(p-1)|s|^{p-2}}{h(x, s)} \geqslant 0$ implies that $H_{x}$ is a strictly convex $\mathrm{C}^{2}$ function and $\mathrm{J}_{\mathrm{x}}$ is a strictly increasing $\mathrm{C}^{1}$ function. Simple calculation shows that

$$
\frac{|s|^{p-1}}{M} \leqslant|J(x, s)| \leqslant \frac{|s|^{p-1}}{m}, \quad \frac{|s|^{p}}{p M} \leqslant H(x, s) \leqslant \frac{|s|^{p}}{p m}, \quad \forall x \in[a, b], s \in \mathbb{R} .
$$

For each $u \in X$, define the functionals $\Phi: X \rightarrow \mathbb{R}$ and $\Psi: X \rightarrow \mathbb{R}$ by

$$
\Phi(u)=\int_{a}^{b} H\left(x, u^{\prime}(x)\right) d x+\frac{\beta}{\alpha} H\left(a, \frac{\alpha}{\beta} u(a)-\frac{1}{\beta} A\right)+\frac{\sigma}{\gamma} H\left(b,-\frac{\gamma}{\sigma} u(b)+\frac{1}{\sigma} B\right)
$$

and

$$
\Psi(u)=\int_{a}^{b} F(x, u(x)) d x+\frac{\mu}{\lambda} \int_{a}^{b} G(x, u(x)) d x,
$$

where $F(x, t):=\int_{0}^{t} f(x, s) d s, G(x, t):=\int_{0}^{t} g(x, s) d s$. In view of (2.1), simple calculation shows that

$$
\begin{aligned}
& \frac{1}{M p}\left(\left\|u^{\prime}\right\|_{L^{p}}^{p}+\frac{\alpha^{p-1}}{\beta^{p-1}}\left|u(a)-\frac{1}{\alpha} A\right|^{p}+\frac{\gamma^{p-1}}{\sigma^{p-1}}\left|u(b)-\frac{1}{\gamma} B\right|^{p}\right) \\
& \leqslant \Phi(u) \leqslant \frac{1}{m p}\left(\left\|u^{\prime}\right\|_{L^{p}}^{p}+\frac{\alpha^{p-1}}{\beta^{p-1}}\left|u(a)-\frac{1}{\alpha} A\right|^{p}+\frac{\gamma^{p-1}}{\sigma^{p-1}}\left|u(b)-\frac{1}{\gamma} B\right|^{p}\right) .
\end{aligned}
$$


Lemma 2.1. Assume that $\mathrm{u} \in \mathrm{X}$ and there exists $\mathrm{r}>0$ such that $\Phi(\mathrm{u}) \leqslant \mathrm{r}$, then

$$
\|\mathrm{u}\|_{\infty} \leqslant(\operatorname{Mpr})^{\frac{1}{p}}\left(\left(\frac{\beta}{\alpha}\right)^{\frac{1}{q}}+(b-a)^{\frac{1}{q}}\right)+\frac{1}{\alpha}|A|,
$$

where $\mathrm{q}$ is the conjugate of $\mathrm{p}$, i.e., $\frac{1}{\mathrm{p}}+\frac{1}{\mathrm{q}}=1$.

Proof. Since $u \in X$, by Theorem 8.2 of [10] and Hölder's inequality, we have

$$
|u(x)|=\left|u(a)+\int_{a}^{x} u^{\prime}(s) d s\right| \leqslant|u(a)|+\int_{a}^{b}\left|u^{\prime}(x)\right| d x \leqslant|u(a)|+\left\|u^{\prime}\right\|_{L^{p}}(b-a)^{\frac{1}{q}} .
$$

Since $\Phi(u) \leqslant r$, from (2.4), we can get

$$
\frac{1}{M p}\left(\left\|u^{\prime}\right\|_{L^{p}}^{p}+\frac{\alpha^{p-1}}{\beta^{p-1}}\left|u(a)-\frac{1}{\alpha} A\right|^{p}+\frac{\gamma^{p-1}}{\sigma^{p-1}}\left|u(b)-\frac{1}{\gamma} B\right|^{p}\right) \leqslant r,
$$

so $\left\|u^{\prime}\right\|_{L^{p}} \leqslant(M p r)^{\frac{1}{p}}$ and

$$
|\mathfrak{u}(\mathrm{a})| \leqslant\left(\frac{\beta}{\alpha}\right)^{\frac{1}{q}}(\mathrm{Mpr})^{\frac{1}{p}}+\frac{1}{\alpha}|A| .
$$

Then in view of (2.5), we have

$$
|u(x)| \leqslant(M p r)^{\frac{1}{p}}\left(\left(\frac{\beta}{\alpha}\right)^{\frac{1}{q}}+(b-a)^{\frac{1}{q}}\right)+\frac{1}{\alpha}|A| .
$$

Hence,

$$
\|u\|_{\infty}=\max _{x \in[a, b]}|u(x)| \leqslant(M p r)^{\frac{1}{p}}\left(\left(\frac{\beta}{\alpha}\right)^{\frac{1}{q}}+(b-a)^{\frac{1}{q}}\right)+\frac{1}{\alpha}|A| .
$$

The proof is complete.

Definition 2.2. We say that $u$ is a classical solution to (1.1) if $u \in C^{1}([a, b]),\left|u^{\prime}\right|^{p-2} u^{\prime} \in A C^{1}([a, b])$, $\alpha u(a)-\beta u^{\prime}(a)=\int_{a}^{b} \xi(x) u(x) d x, \gamma u(b)+\sigma u^{\prime}(b)=\int_{a}^{b} \eta(x) u(x) d x$, and

$$
-\left(\phi_{\mathfrak{p}}\left(\mathfrak{u}^{\prime}(x)\right)\right)^{\prime}=\left(\lambda f(x, u(x))+\mu g(x, u(x))+\int_{0}^{\mathfrak{u}^{\prime}(x)} \frac{\partial}{\partial x}\left(\frac{(p-1)|\tau|^{p-2}}{h(x, \tau)}\right) d \tau\right) h\left(x, u^{\prime}(x)\right)
$$

for almost every $x \in[a, b]$.

Definition 2.3. We say that $u$ is a weak solution to (1.1) if $u \in X$ and

$$
\begin{aligned}
& \int_{a}^{b} J\left(x, u^{\prime}(x)\right) v^{\prime}(x) d x+J\left(a, \frac{\alpha}{\beta} u(a)-\frac{1}{\beta} A\right) v(a)-J\left(b,-\frac{\gamma}{\sigma} u(b)+\frac{1}{\sigma} B\right) v(b) \\
& \quad=\lambda \int_{a}^{b} f(x, u(x)) v(x) d x+\mu \int_{a}^{b} g(x, u(x)) v(x) d x
\end{aligned}
$$

for any $v \in X$.

The proofs of the next two lemmas are similar to the argument in [28] with minor changes. For the readers' convenience, we present the proofs in detail.

Lemma 2.4. The classical and weak solutions to (1.1) coincide. 
Proof. Assume that $u$ is a classical solution to (1.1), it is obvious that $u \in W^{1, p}([a, b])$.

Since $\inf _{(x, t) \in[a, b] \times \mathbb{R}} h(x, t)>0$, we have

$$
\begin{aligned}
0 & =-\frac{\left(\phi_{p}\left(u^{\prime}(x)\right)\right)^{\prime}}{h\left(x, u^{\prime}(x)\right)}-\int_{0}^{u^{\prime}(x)} \frac{\partial}{\partial x}\left(\frac{(p-1)|\tau|^{p-2}}{h(x, \tau)}\right) d \tau-\lambda f(x, u(x))-\mu g(x, u(x)) \\
& =-\frac{d}{d x}\left(\int_{0}^{u^{\prime}(x)} \frac{(p-1)|\tau|^{p-2}}{h(x, \tau)} d \tau\right)-\lambda f(x, u(x))-\mu g(x, u(x)) .
\end{aligned}
$$

Multiplying (2.6) by any $v \in W^{1, p}([a, b])$, and then integrating it over $[a, b]$, we have

$$
\begin{aligned}
0= & -\int_{a}^{b} \frac{d}{d x}\left(\int_{0}^{u^{\prime}(x)} \frac{(p-1)|\tau|^{p-2}}{h(x, \tau)} d \tau\right) v(x) d x-\lambda \int_{a}^{b} f(x, u(x)) v(x) d x-\mu \int_{a}^{b} g(x, u(x)) v(x) d x \\
= & \int_{a}^{b} J\left(x, u^{\prime}(x)\right) v^{\prime}(x) d x+J\left(a, u^{\prime}(a)\right) v(a)-J\left(b, u^{\prime}(b)\right) v(b) \\
& -\lambda \int_{a}^{b} f(x, u(x)) v(x) d x-\mu \int_{a}^{b} g(x, u(x)) v(x) d x, \\
= & \int_{a}^{b} J\left(x, u^{\prime}(x)\right) v^{\prime}(x) d x+J\left(a, \frac{\alpha}{\beta} u(a)-\frac{1}{\beta} A\right) v(a)-J\left(b,-\frac{\gamma}{\sigma} u(b)+\frac{1}{\sigma} B\right) v(b) \\
& -\lambda \int_{a}^{b} f(x, u(x)) v(x) d x-\mu \int_{a}^{b} g(x, u(x)) v(x) d x,
\end{aligned}
$$

which means that $u$ is a weak solution to (1.1). On the other hand, if $u$ is a weak solution to (1.1), by integration by parts on $[a, b]$, we have

$$
\begin{aligned}
0= & \int_{a}^{b} J\left(x, u^{\prime}(x)\right) v^{\prime}(x) d x+J\left(a, \frac{\alpha}{\beta} u(a)-\frac{1}{\beta} A\right) v(a)-J\left(b,-\frac{\gamma}{\sigma} u(b)+\frac{1}{\sigma} B\right) v(b) \\
& -\lambda \int_{a}^{b} f(x, u(x)) v(x) d x-\mu \int_{a}^{b} g(x, u(x)) v(x) d x \\
= & J\left(b, u^{\prime}(b)\right) v(b)-J\left(a, u^{\prime}(a)\right) v(a)-\int_{a}^{b} \frac{d}{d x}\left(J\left(x, u^{\prime}(x)\right)\right) v(x) d x \\
& +J\left(a, \frac{\alpha}{\beta} u(a)-\frac{1}{\beta} A\right) v(a)-J\left(b,-\frac{\gamma}{\sigma} u(b)+\frac{1}{\sigma} B\right) v(b) \\
& -\lambda \int_{a}^{b} f(x, u(x)) v(x) d x-\mu \int_{a}^{b} g(x, u(x)) v(x) d x
\end{aligned}
$$

i.e.,

$$
\begin{aligned}
0= & -\int_{a}^{b}\left\{\frac{\left(\phi_{p}\left(u^{\prime}(x)\right)\right)^{\prime}}{h\left(x, u^{\prime}(x)\right)}+\lambda f(x, u(x))+\mu g(x, u(x))+\int_{0}^{u^{\prime}(x)} \frac{\partial}{\partial x}\left(\frac{(p-1)|\tau|^{p-2}}{h(x, \tau)}\right) d \tau\right\} v(x) d x \\
& \times\left[J\left(b, u^{\prime}(b)\right)-J\left(b,-\frac{\gamma}{\sigma} u(b)+\frac{1}{\sigma} B\right)\right] v(b)+\left[-J\left(a, u^{\prime}(a)\right)+J\left(a, \frac{\alpha}{\beta} u(a)-\frac{1}{\beta} A\right)\right] v(a)
\end{aligned}
$$

holds for any $v \in W^{1, q}([a, b])$, and hence holds for any $v \in C_{0}^{\infty}([a, b])$. By the fundamental lemma of variational, $u$ satisfies equation (2.6) for a.e. $x \in[a, b]$, and therefore (2.7) becomes

$$
0=\left[J\left(b, u^{\prime}(b)\right)-J\left(b,-\frac{\gamma}{\sigma} u(b)+\frac{1}{\sigma} B\right)\right] v(b)+\left[-J\left(a, u^{\prime}(a)\right)+J\left(a, \frac{\alpha}{\beta} u(a)-\frac{1}{\beta} A\right)\right] v(a)
$$


for any $v \in W^{1, p}([a, b])$. We claim that $u$ satisfies the boundary conditions in (1.1). Otherwise, without loss of generality, assume that

$$
\gamma u(b)+\sigma u^{\prime}(b)>B .
$$

Since $\sigma>0$ and $\mathrm{J}_{\mathrm{b}}(\mathrm{s})=\mathrm{J}(\mathrm{b}, \mathrm{s})$ is strictly increasing, we get that

$$
J\left(b, u^{\prime}(b)\right)-J\left(b,-\frac{\gamma}{\sigma} u(b)+\frac{1}{\sigma} B\right)>0 .
$$

Let $v(x)=x-a$, then $v \in C^{\infty}([a, b]) \subset W^{1, p}([a, b])$ and

$$
\begin{aligned}
0 & =\left[J\left(b, u^{\prime}(b)\right)-J\left(b,-\frac{\gamma}{\sigma} u(b)+\frac{1}{\sigma} B\right)\right] v(b)+\left[-J\left(a, u^{\prime}(a)\right)+J\left(a, \frac{\alpha}{\beta} u(a)-\frac{1}{\beta} A\right)\right] v(a) \\
& =\left[J\left(b, u^{\prime}(b)\right)-J\left(b,-\frac{\gamma}{\sigma} u(b)+\frac{1}{\sigma} B\right)\right](b-a)+0>0,
\end{aligned}
$$

which is a contradiction. Therefore, $u$ is a classical solution to (1.1).

Lemma 2.5. Assume that the functionals $\Psi, \Phi: X \rightarrow \mathbb{R}$ are defined by (2.2) and (2.3), respectively. Then

(a) $\Phi$ is sequentially weakly lower semicontinuous, continuous, $\lim _{\|u\|+\infty} \Phi(\mathfrak{u})=+\infty$ and its Gâteaux derivative at the point $\mathrm{u} \in \mathrm{X}$ is the functional $\Phi^{\prime}(\mathrm{u})$ given by

$$
\Phi^{\prime}(u)(v)=\int_{a}^{b} J\left(x, u^{\prime}(x)\right) v^{\prime}(x) d x+J\left(a, \frac{\alpha}{\beta} u(a)-\frac{1}{\beta} A\right) v(a)-J\left(b,-\frac{\gamma}{\sigma} u(b)+\frac{1}{\sigma} B\right) v(b)
$$

for every $v \in X$;

(b) $\Psi$ is sequentially weakly lower continuous and its Gâteaux derivative at the point $\mathrm{u} \in \mathrm{X}$ is the functional $\Psi^{\prime}(u)$ given by

$$
\Psi^{\prime}(u)(v)=\int_{a}^{b} f(x, u(x)) v(x) d x+\frac{\mu}{\lambda} \int_{a}^{b} g(x, u(x)) v(x) d x
$$

for every $v \in X$.

Proof. Assume that $\left\{u_{n}\right\} \subset X$ and $u_{n} \rightarrow u$ in $W^{1, p}([a, b])$ as $n \rightarrow \infty$, then $\max \left\{\left\|u_{n}-u\right\|_{L^{p}},\left\|u_{n}^{\prime}-u^{\prime}\right\|_{L^{p}}\right\} \leqslant$ $\left\|u_{n}-u\right\| \rightarrow 0$ as $n \rightarrow \infty$. Since the Sobolev space $W^{1, p}([a, b])$ is compactly embedded into $C([a, b])$, we have $u_{n}(a) \rightarrow u(a), u_{n}(b) \rightarrow u(b)$ as $n \rightarrow \infty$. So

$$
\begin{aligned}
\left|\Phi\left(u_{n}\right)-\Phi(u)\right| \leqslant & \int_{a}^{b}\left|H\left(x, u_{n}^{\prime}(x)\right)-H\left(x, u^{\prime}(x)\right)\right| d x \\
& +\frac{\beta}{\alpha}\left|H\left(a, \frac{\alpha}{\beta} u_{n}(a)-\frac{1}{\beta} A\right)-H\left(a, \frac{\alpha}{\beta} u(a)-\frac{1}{\beta} A\right)\right| \\
& +\frac{\sigma}{\gamma}\left|H\left(b,-\frac{\gamma}{\sigma} u_{n}(b)+\frac{1}{\sigma} B\right)-H\left(b,-\frac{\gamma}{\sigma} u(b)+\frac{1}{\sigma} B\right)\right| \\
= & : I_{1}+I_{2}+I_{3} .
\end{aligned}
$$

By the mean value theorem, there exists a function $\theta(x)$ such that $0<\theta(x)<1$ and

$$
I_{1}=\int_{a}^{b}\left|H\left(x, u_{n}^{\prime}(x)\right)-H\left(x, u^{\prime}(x)\right)\right| d x=\int_{a}^{b}\left|J\left(x, u^{\prime}(x)+\theta(x)\left(u_{n}^{\prime}(x)-u^{\prime}(x)\right)\right)\left(u_{n}^{\prime}(x)-u^{\prime}(x)\right)\right| d x,
$$

then in view of (2.1) we have

$$
I_{1} \leqslant \frac{1}{m} \int_{a}^{b}\left|u^{\prime}(x)+\theta(x)\left(u_{n}^{\prime}(x)-u^{\prime}(x)\right)\right|^{p-1}\left|u_{n}^{\prime}(x)-u^{\prime}(x)\right| d x
$$




$$
\begin{aligned}
& \leqslant \frac{2^{p-1}}{m} \int_{a}^{b}\left(\left|u^{\prime}(x)\right|^{p-1}+|\theta(x)|^{p-1}\left|u_{n}^{\prime}(x)-u^{\prime}(x)\right|^{p-1}\right)\left|u_{n}^{\prime}(x)-u^{\prime}(x)\right| d x \\
& \leqslant \frac{2^{p-1}}{m} \int_{a}^{b}\left(\left|u^{\prime}(x)\right|^{p-1}\left|u_{n}^{\prime}(x)-u^{\prime}(x)\right|+\left|u_{n}^{\prime}(x)-u^{\prime}(x)\right|^{p}\right) d x \\
& \leqslant \frac{2^{p-1}}{m}\left(\left\|u^{\prime}\right\|_{L^{p}}^{\frac{p}{q}}\left\|u_{n}^{\prime}-u^{\prime}\right\|_{L^{p}}+\left\|u_{n}^{\prime}-u^{\prime}\right\|_{L^{p}}^{p}\right) \\
& \rightarrow 0 \text { as } n \rightarrow \infty .
\end{aligned}
$$

By the continuity of $\mathrm{H}(x, s)$ and $\mathrm{u}_{n}(\mathrm{a}) \rightarrow \mathrm{u}(\mathrm{a}), \mathrm{u}_{n}(\mathrm{~b}) \rightarrow \mathrm{u}(\mathrm{b}),\left\|\mathrm{u}_{n}-\mathrm{u}\right\|_{\mathrm{L}^{p}} \rightarrow 0$ as $n \rightarrow \infty$, we have $\mathrm{I}_{2}, \mathrm{I}_{3} \rightarrow 0$ as $\mathrm{n} \rightarrow \infty$. Thus we have shown that $\Phi$ is continuous.

Since $H_{x}(s)$ is a strictly convex function for any $x \in[a, b]$, it is easy to verify that $\Phi$ is a strictly convex functional, so from Proposition 25.20 of [31], we obtain that $\Phi$ is a sequentially weakly lower semicontinuous functional.

To prove the coercivity of $\Phi$, we assume that $\|u\| \rightarrow+\infty$ and consider the two cases: (i) $\left\|u^{\prime}\right\|_{L^{p}} \rightarrow+\infty$, (ii) $\|\mathrm{u}\|_{\mathrm{L}^{p}} \rightarrow+\infty$ while $\left\|\mathrm{u}^{\prime}\right\|_{\mathrm{L}^{p}} \not \rightarrow+\infty$.

(i) By the estimate (2.4), we obtain that $\Phi(u) \geqslant \frac{1}{M p}\left\|u^{\prime}\right\|_{L^{p}}^{p} \rightarrow+\infty$ as $\|u\| \rightarrow+\infty$.

(ii) Since $W^{1, p}([a, b])$ is continuously embedded into $C([a, b])$, we have $u \in L^{\infty}([a, b])$ and $\|u\|_{L^{p}} \leqslant$ $(b-a)^{\frac{1}{p}}\|u\|_{\infty}$, hence $\|u\|_{\infty} \rightarrow+\infty$ as $\|\mathfrak{u}\|_{L^{p}} \rightarrow \infty$. From the proof of Lemma 2.1, we can infer that

$$
|u(a)| \geqslant\|u\|_{\infty}-\left\|u^{\prime}\right\|_{L^{p}}(b-a)^{\frac{1}{q}} \rightarrow+\infty
$$

as $\|\mathrm{u}\|_{\infty} \rightarrow+\infty,\left\|\mathrm{u}^{\prime}\right\|_{\mathrm{L}^{\mathrm{p}}} \not \rightarrow+\infty$. In view of the estimate (2.4), we have

$$
\Phi(\mathrm{u}) \geqslant \frac{1}{M p} \frac{\alpha^{p-1}}{\beta^{p-1}}\left|u(a)-\frac{1}{\alpha} A\right|^{p} \rightarrow+\infty,
$$

as $\|\mathrm{u}\|_{\infty} \rightarrow+\infty,\left\|\mathrm{u}^{\prime}\right\|_{\mathrm{L}^{\mathrm{p}}} \not \rightarrow \infty$.

In conclusion, $\Phi$ is coercive.

By the definition of the Gâteaux derivative, it is easy to verify that

$$
\Phi^{\prime}(u)(v)=\int_{a}^{b} J\left(x, u^{\prime}(x)\right) v^{\prime}(x) d x+J\left(a, \frac{\alpha}{\beta} u(a)-\frac{1}{\beta} A\right) v(a)-J\left(b,-\frac{\gamma}{\sigma} u(b)+\frac{1}{\sigma} B\right) v(b)
$$

for every $v \in X$. The proof of part (a) is complete.

Part (b) follows from standard arguments, and thus we omit the details.

Remark 2.6. Following from Definition 2.3, Lemma 2.4, and Lemma 2.6, we get that $u \in X$ is a critical point of $I_{\lambda}:=\Phi-\lambda \Psi$ if and only if $u$ is a classical solution to BVP (1.1) for some $\lambda>0, \mu \geqslant 0$.

Now we recall Ricceri's Variational Principle.

Lemma 2.7 ([23]). Let $X$ be a real reflexive Banach space and let $\Psi, \Phi: X \rightarrow \mathbb{R}$ be two Gâteaux differentiable functionals such that $\Psi$ is sequentially weakly lower semicontinuous, strongly continuous and coercive, and $\Psi$ is sequentially weakly continuous. For every $r>\inf _{X} \Phi$, let

and

$$
\phi(r):=\inf _{u \in \Phi^{-1}(-\infty, r)} \frac{\sup _{v \in \Phi^{-1}(-\infty, r)} \Psi(v)-\Psi(u)}{r-\Phi(u)}
$$

$$
\zeta:=\liminf _{r \rightarrow+\infty} \phi(r), \quad \delta:=\liminf _{r \rightarrow\left(\inf _{X} \Phi\right)^{+}} \phi(r) .
$$

Then

(a) for every $r>\inf _{X} \Phi$ and every $\lambda \in\left(0, \frac{1}{\phi(r)}\right)$, the restriction of the functional $I_{\lambda}:=\Phi-\lambda \Psi$ to $\Phi^{-1}(-\infty, r)$ admits a global minimum, which is a critical point (local minima) of $\mathrm{I}_{\lambda}$ in $\mathrm{X}$; 
(b) if $\zeta<+\infty$, then for each $\lambda \in\left(0, \frac{1}{\zeta}\right)$ the following alternative holds: either

$\left(\mathrm{b}_{1}\right) \mathrm{I}_{\lambda}$ possesses a global minimum, or

$\left(b_{2}\right)$ there is a sequence $\left\{u_{n}\right\}$ of critical points (local minima) of $\mathrm{I}_{\lambda}$ such that $\lim _{n \rightarrow \infty} \Phi\left(u_{n}\right)=\infty$;

(c) if $\delta<+\infty$, then for each $\lambda \in\left(0, \frac{1}{\delta}\right)$ the following alternative holds: either

$\left(c_{1}\right)$ there is a global minimum of $\Phi$ which is a local minimum of $\mathrm{I}_{\lambda}$, or

$\left(c_{2}\right)$ there is a sequence $\left\{u_{n}\right\}$ of pairwise distinct critical points (local minima) of $\mathrm{I}_{\lambda}$ that converges weakly to a global minimum of $\Phi$.

\section{Main result}

For any $v>0$, we define

$$
\mathrm{Q}(v):=\left\{t \in \mathbb{R}:|t| \leqslant v\left(\left(\frac{\beta}{\alpha}\right)^{\frac{1}{q}}+(b-a)^{\frac{1}{q}}\right)+\frac{1}{\alpha}|A|\right\} .
$$

Theorem 3.1. Assume that

$\left(\mathrm{H}_{1}\right)$

$$
A_{\infty}:=\liminf _{v \rightarrow+\infty} \frac{\int_{a}^{b} \sup _{t \in Q(v)} F(x, t) d x}{v^{p}-M p \Phi(0)}<\frac{m}{M} \limsup _{t \rightarrow+\infty} \frac{\int_{a}^{b} F(x, t) d x}{\left(\frac{\alpha}{\beta}\right)^{p-1}|t-A|^{p}+\left(\frac{\gamma}{\sigma}\right)^{p-1}|t-B|^{p}}
$$

Then for every $\lambda \in \Lambda$, where

$$
\Lambda=\left(\frac{1}{\operatorname{mp}_{\limsup \sup _{t \rightarrow \infty} \frac{\int_{a}^{b} F(x, t) d x}{\left(\frac{\alpha}{\beta}\right)^{p-1}|t-A|^{p}+\left(\frac{\gamma}{\sigma}\right)^{p-1}|t-B|^{p}}}}, \frac{1}{\operatorname{Mp} \liminf _{v \rightarrow+\infty} \frac{\int_{a}^{b} \sup _{t \in Q(v)} F(x, t) d x}{v^{p}-M p \Phi(0)}}\right),
$$

and for every $\mathrm{L}^{1}$-Carathéodary function $\mathrm{g}:[\mathrm{a}, \mathrm{b}] \times \mathbb{R} \rightarrow \mathbb{R}$ satisfying:

$\left(\mathrm{H}_{2}\right)$

$$
0 \leqslant B_{\infty}:=\liminf _{v \rightarrow+\infty} \frac{\int_{a}^{b} \sup _{t \in Q(v)} G(x, t) d x}{v^{p}-\operatorname{Mp} \Phi(0)}<+\infty
$$

$\left(\mathrm{H}_{3}\right)$

$$
\liminf _{t \rightarrow+\infty} \int_{a}^{b} G(x, t) d x>-\infty
$$

there exists $\delta_{g, \lambda}:=\frac{1}{\mathrm{~B}_{\infty}}\left(\frac{1}{M p}-\lambda \mathrm{A}_{\infty}\right)>0$, such that for any $\mu \in\left[0, \delta_{g, \lambda}\right)$, the boundary value problem (1.1) has an unbounded sequence of classical solutions in $\mathrm{X}$.

Proof. Let the functionals $\Phi$ and $\Psi$ be defined by (2.2) and (2.3), respectively. From Lemma 2.5 we obtain that $\Phi$ and $\Psi$ satisfy all the regularity assumptions given in Lemma 2.7. In addition, by (2.3) and (2.4) it is easy to verify that $\Phi(0)=\frac{\beta}{\alpha} H\left(a,-\frac{1}{\beta} A\right)+\frac{\sigma}{\gamma} H\left(b, \frac{1}{\sigma} B\right) \geqslant 0$ and $\Psi(0)=0$ for any $\lambda>0$ and $\mu \geqslant 0$. For any $\lambda \in \Lambda$, we have $\frac{1}{M p}-\lambda A_{\infty}>0$. 
First, we claim that $\zeta<+\infty$. To prove this, let $\left\{v_{k}\right\}$ be a sequence of positive numbers such that $v_{k} \rightarrow+\infty$ as $k \rightarrow \infty$ and

$$
\liminf _{k \rightarrow \infty} \frac{\int_{a}^{b} \sup _{t \in Q\left(v_{k}\right)}\left(F(x, t)+\frac{\mu}{\lambda} G(x, t)\right) d x}{v_{k}^{p}-M p \Phi(0)}=\liminf _{v \rightarrow+\infty} \frac{\int_{a}^{b} \sup _{t \in Q(v)}\left(F(x, t)+\frac{\mu}{\lambda} G(x, t)\right) d x}{v^{p}-M p \Phi(0)} .
$$

Let $r_{k}=\frac{v_{k}^{p}}{M p}$ for each $k \in \mathbb{N}$, then

$$
\begin{aligned}
\zeta=\liminf _{r \rightarrow+\infty} \phi(r) \leqslant \liminf _{k \rightarrow \infty} \phi\left(r_{k}\right) & =\liminf _{k \rightarrow \infty} \inf _{u \in \Phi^{-1}\left(-\infty, r_{k}\right)} \frac{\sup _{v \in \Phi^{-1}\left(-\infty, r_{k}\right)} \Psi(v)-\Psi(u)}{r_{k}-\Phi(u)} \\
& \leqslant \liminf _{k \rightarrow \infty} \frac{\sup _{v \in \Phi^{-1}\left(-\infty, r_{k}\right)} \Psi(v)-\Psi(0)}{r_{k}-\Phi(0)} \\
& \leqslant \liminf _{k \rightarrow \infty} \frac{\sup _{v \in \Phi^{-1}\left(-\infty, r_{k}\right)} \Psi(v)}{r_{k}-\Phi(0)} .
\end{aligned}
$$

By Lemma 2.1, we have

$$
\begin{aligned}
\Phi^{-1}\left(-\infty, r_{k}\right) & =\left\{u \in X: \Phi(u) \leqslant r_{k}\right\} \\
& \subset\left\{u \in X:\|u\|_{\infty} \leqslant\left(M p r_{k}\right)^{\frac{1}{p}}\left(\left(\frac{\beta}{\alpha}\right)^{\frac{1}{q}}+(b-a)^{\frac{1}{q}}\right)+\frac{1}{\alpha}|A|\right\} \\
& =\left\{u \in X: \max _{x \in[a, b]}|u(x)| \in Q\left(\left(M p r_{k}\right)^{\frac{1}{p}}\right)\right\} .
\end{aligned}
$$

Then in view of (3.1), (3.3), (3.4), $\left(\mathrm{H}_{1}\right)$, and $\left(\mathrm{H}_{2}\right)$, we obtain that

$$
\begin{aligned}
\zeta & \leqslant M p \liminf _{k \rightarrow \infty} \frac{\int_{a}^{b} \sup _{t \in Q\left(v_{k}\right)}\left(F(x, t)+\frac{\mu}{\lambda} G(x, t)\right) d x}{v_{k}^{p}-M p \Phi(0)} \\
& \leqslant M p\left(\liminf _{v \rightarrow+\infty} \frac{\int_{a}^{b} \sup _{t \in Q(v)} F(x, t) d x}{v^{p}-M p \Phi(0)}+\frac{\mu}{\lambda} \liminf _{v \rightarrow+\infty} \frac{\int_{a}^{b} \sup _{t \in Q(v)} G(x, t) d x}{v^{p}-M p \Phi(0)}\right) \\
& =M p\left(A_{\infty}+\frac{\mu}{\lambda} B_{\infty}\right)<+\infty .
\end{aligned}
$$

Since $\mu \in\left[0, \delta_{g, \lambda}\right)$, in view of (3.5), we have $\zeta \leqslant M p\left(A_{\infty}+\frac{\mu}{\lambda} B_{\infty}\right)<\frac{1}{\lambda}$, i.e., $\lambda<\frac{1}{\zeta}$. Hence $\Lambda \subset\left(0, \frac{1}{\zeta}\right)$. For any fixed $\lambda \in \Lambda$, by Lemma 2.7 (b), one of the following alternatives holds:

$\left(b_{1}\right) I_{\lambda}$ possesses a global minimum, or

$\left(b_{2}\right)$ there is a sequence $\left\{u_{n}\right\}$ of critical points (local minima) of $I_{\lambda}$ such that $\lim _{n \rightarrow \infty} \Phi\left(u_{n}\right)=\infty$.

Now we show that the functional $I_{\lambda}$ is unbounded from below, hence the alternative $\left(b_{1}\right)$ does not hold.

Let $\left\{d_{k}\right\}$ be a sequence of positive numbers such that $d_{k} \rightarrow+\infty$, as $k \rightarrow \infty$, and define a sequence of functions $\left\{w_{k}\right\}$ such that $w_{k}(x) \equiv d_{k}$ for all $x \in[a, b]$, then $w_{k} \in X$. From (2.3) and (2.4) we have

$$
\begin{aligned}
\mathrm{I}_{\lambda}\left(w_{\mathrm{k}}\right) & =\Phi\left(w_{k}\right)-\lambda \Psi\left(w_{k}\right) \\
& \leqslant \frac{1}{m p}\left(\left(\frac{\alpha}{\beta}\right)^{p-1}\left|d_{k}-A\right|^{p}+\left(\frac{\gamma}{\sigma}\right)^{p-1}\left|d_{k}-B\right|^{p}\right)-\lambda \int_{a}^{b} F\left(x, d_{k}\right) d x-\mu \int_{a}^{b} G\left(x, d_{k}\right) d x .
\end{aligned}
$$

Since $\lambda \in \Lambda$, (3.2) implies that

$$
\frac{1}{\lambda}<m p \limsup _{t \rightarrow+\infty} \frac{\int_{a}^{b} F(x, t) d x}{\left.\left(\frac{\alpha}{\beta}\right)^{p-1}|t-A|^{p}+\left(\frac{\gamma}{\sigma}\right)^{p-1}|t-B|^{p}\right)} .
$$


Then there exist $\varepsilon>0$ and $N \in \mathbb{N}$, such that

$$
\frac{1}{\lambda}<\varepsilon<\frac{\int_{a}^{b} F\left(x, d_{k}\right) d x}{\frac{1}{m p}\left(\left(\frac{\alpha}{\beta}\right)^{p-1}\left|d_{k}-A\right|^{p}+\left(\frac{\gamma}{\sigma}\right)^{p-1}\left|d_{k}-B\right|^{p}\right)}
$$

for every $k \geqslant N$, hence $1-\lambda \varepsilon<0$ and

$$
\lambda \varepsilon \frac{1}{m p}\left(\left(\frac{\alpha}{\beta}\right)^{p-1}\left|d_{k}-A\right|^{p}+\left(\frac{\gamma}{\sigma}\right)^{p-1}\left|d_{k}-B\right|^{p}\right)<\lambda \int_{a}^{b} F\left(x, d_{k}\right) d x
$$

for every $k \geqslant N$. In view of $\left(H_{3}\right)$, we have

$$
\mu \liminf _{k \rightarrow \infty} \int_{a}^{b} G\left(x, d_{k}\right) d x>-\infty .
$$

From (3.6)-(3.8) we obtain that

$$
\mathrm{I}_{k}\left(w_{k}\right)<(1-\lambda \varepsilon) \frac{1}{m p}\left(\left(\frac{\alpha}{\beta}\right)^{p-1}\left|\mathrm{~d}_{k}-A\right|^{p}+\left(\frac{\gamma}{\sigma}\right)^{p-1}\left|\mathrm{~d}_{k}-\mathrm{B}\right|^{p}\right)-\mu \int_{a}^{b} \mathrm{G}\left(x, \mathrm{~d}_{k}\right) \mathrm{d} x \rightarrow-\infty
$$

as $k \rightarrow \infty$, that is, the functional $I_{\lambda}$ is unbounded from below. Therefore, by $\left(b_{2}\right)$ in Lemma 2.7 , there exists a sequence $\left\{u_{k}\right\}$ of critical points of $I_{\lambda}$ such that $\lim _{k \rightarrow \infty} \Phi\left(u_{k}\right)=+\infty$. In view of (2.4), we have $\left\|\mathfrak{u}_{k}\right\| \rightarrow+\infty$. Finally, taking Remark 2.6 into account completes the proof of the theorem.

Let $h(x, t) \equiv h(t)$ and $A=B=0$, then $\frac{\partial}{\partial x}\left(\frac{(p-1)|\tau|^{p-2}}{h(x, \tau)}\right)=0$ and $\Phi(0)=0$, and we have the following special case of Theorem 3.1.

Corollary 3.2. Assume that

$$
A_{\infty}=\liminf _{v \rightarrow+\infty} \frac{\int_{a}^{b} \sup _{t \in Q_{1}(v)} F(x, t) d x}{v^{p}}<\frac{m}{M} \limsup _{t \rightarrow+\infty} \frac{\int_{a}^{b} F(x, t) d x}{\left(\left(\frac{\alpha}{\beta}\right)^{p-1}+\left(\frac{\gamma}{\sigma}\right)^{p-1}\right) t^{p}},
$$

where

$$
\mathrm{Q}_{1}(v)=\left\{t \in \mathbb{R}:|t| \leqslant v\left(\left(\frac{\beta}{\alpha}\right)^{\frac{1}{q}}+(b-a)^{\frac{1}{q}}\right)\right\}
$$

Then for each

$$
\lambda \in\left(\frac{1}{\operatorname{mp}_{\limsup \sup \frac{\int_{a}^{b} F(x, t) d x}{\left(\left(\frac{\alpha}{\beta}\right)^{p-1}+\left(\frac{\gamma}{\sigma}\right)^{p-1}\right)^{p}}}}, \frac{1}{M p \liminf _{\gamma \rightarrow+\infty} \frac{\int_{a}^{b} \sup _{t \in Q_{1}(v)} F(x, t) d x}{\nu^{p}}}\right)
$$

and for every $\mathrm{L}^{1}$-Carathéodary function $\mathrm{g}:[\mathrm{a}, \mathrm{b}] \times \mathbb{R} \rightarrow \mathbb{R}$ satisfying $\left(\mathrm{H}_{2}\right)$ and $\left(\mathrm{H}_{3}\right)$, there exists $\delta_{\mathrm{g}, \lambda}:=$ $\frac{1}{\mathrm{~B}_{\infty}}\left(\frac{1}{\mathrm{Mp}}-\lambda \mathrm{A}_{\infty}\right)>0$, such that for any $\mu \in\left[0, \delta_{g, \lambda}\right)$, the problem

$$
\left\{\begin{array}{l}
-\left(\phi_{p}\left(u^{\prime}\right)\right)^{\prime}=(\lambda f(x, u(x))+\mu g(x, u(x))) h\left(u^{\prime}(x)\right), \quad x \in(a, b), \\
\alpha u(a)-\beta u^{\prime}(a)=0, \quad \gamma u(b)+\sigma u^{\prime}(b)=0
\end{array}\right.
$$

has an unbounded sequence of classical solutions in $\mathrm{X}$.

Remark 3.3. Let $g(x, t) \equiv 0$, by Corollary 3.2, we can obtain the scalar case of Theorem 3.1 of [18].

Moreover, assume that $f(x, t) \equiv f(t), g(x, t) \equiv g(t), \alpha=\beta=\gamma=\eta=1$, we have the following special case of Corollary 3.2. 
Corollary 3.4. Assume that

$$
\liminf _{v \rightarrow+\infty} \frac{\max _{t \in Q_{2}(v)} F(t)}{v^{p}}=0, \quad \limsup _{t \rightarrow+\infty} \frac{F(t)}{t^{p}}=+\infty,
$$

where $\mathrm{Q}_{2}(v)=\left\{t \in \mathbb{R}:|t| \leqslant v\left(1+(b-a)^{\frac{1}{q}}\right)\right\}$. Then for every $\lambda \in(0,+\infty), \mu \in[0,+\infty)$ and for every continuous function $\mathrm{g}: \mathbb{R} \rightarrow \mathbb{R}$ which satisfies

$$
\liminf _{v \rightarrow+\infty} \frac{\max _{t \in Q_{2}(v)} G(t)}{v^{p}}=0 \text { and } \liminf _{t \rightarrow+\infty} G(t)>-\infty,
$$

the boundary value problem

$$
\left\{\begin{array}{l}
-\left(\phi_{p}\left(u^{\prime}\right)\right)^{\prime}=(\lambda f(u(x))+\mu g(u(x))) h\left(u^{\prime}(x)\right), \quad x \in(a, b), \\
u(a)-u^{\prime}(a)=0, \quad u(b)+u^{\prime}(b)=0
\end{array}\right.
$$

has an unbounded sequence of classical solutions in $\mathrm{X}$.

Since $X$ is a real reflexive Banach space, $\Phi$ is a strictly convex and coercive functional on $X$ and $\inf _{X} \Phi \geqslant 0$, we know that $\Phi$ has a unique global minimum $\mathfrak{u}_{0} \in X$ such that $\Phi\left(u_{0}\right)=\inf _{X} \Phi \geqslant 0$. Using (c) in Lemma 2.7 and the argument as in the proof of Theorem 3.1, we can obtain the following result.

Theorem 3.5. Assume that

$$
\begin{aligned}
A_{0} & :=\liminf _{v \rightarrow\left(\left(M p \inf _{x} \Phi\right)^{\frac{1}{p}}\right)^{+}} \frac{\sup _{t \in Q(v)} F(x, t) d x}{v^{p}-M p \Phi(0)} \\
< & \frac{m}{M} \limsup _{u \rightarrow u_{0}} \frac{\int_{a}^{b} F(x, u(x)) d x}{\left\|u^{\prime}\right\|_{L^{p}}^{p}+\left(\frac{\alpha}{\beta}\right)^{p-1}|u(a)-A|^{p}+\left(\frac{\gamma}{\sigma}\right)^{p-1}|u(b)-B|^{p}} .
\end{aligned}
$$

Then, for each $\lambda \in \Lambda^{\prime}$, where

$$
\begin{aligned}
& \Lambda^{\prime}=\left(\frac{1}{\operatorname{mp}_{\limsup _{u} \operatorname{uup}_{0}} \frac{\int_{a}^{b} F(x, t) d x}{\left\|u^{\prime}\right\|_{L^{p}}^{p}+\left(\frac{\alpha}{\beta}\right)^{p-1}|u(a)-A|^{p}+\left(\frac{\gamma}{\sigma}\right)^{p-1}|u(b)-B|^{p}}},\right. \\
& \left.\frac{1}{\operatorname{Mp}_{v \rightarrow\left(\left(M p \inf _{X} \Phi\right)^{\frac{1}{p}}\right)^{+}} \frac{\sup _{\mathrm{t} \in \mathrm{Q}(v)} \mathrm{F}(x, \mathrm{t}) \mathrm{d} x}{v^{p}-M p \Phi(0)}}\right)
\end{aligned}
$$

and for every $\mathrm{L}^{1}$-Carathéodary function $\mathrm{g}:[\mathrm{a}, \mathrm{b}] \times \mathbb{R} \rightarrow \mathbb{R}$ satisfying:

$\left(\mathrm{H}_{2}^{\prime}\right)$

$$
0 \leqslant B_{0}:=\liminf _{v \rightarrow\left(\left(M p \inf _{X} \Phi\right)^{\frac{1}{p}}\right)^{+}} \frac{\int_{a}^{b} \sup _{t \in Q(v)} G(x, t) d x}{v^{p}-M p \Phi(0)}<+\infty
$$

$\left(\mathrm{H}_{3}^{\prime}\right)$

$$
\liminf _{\mathfrak{u} \rightarrow u_{0}} \int_{a}^{b} G(x, u(x)) d x \geqslant-\inf _{x} \Phi
$$


there exists $\delta_{g, \lambda}^{\prime}:=\frac{1}{\mathrm{~B}_{0}}\left(\frac{1}{\mathrm{Mp}}-\lambda \mathrm{A}_{0}\right)>0$, such that for any $\mu \in\left[0, \delta_{g, \lambda}^{\prime}\right)$, the boundary value problem (1.1) has a sequence of classical solutions in $X$ converging uniformly to $\mathrm{u}_{0}$.

Proof. Let the functionals $\Phi$ and $\Psi$ be defined by (2.2) and (2.3), respectively. From Lemma 2.5 we obtain that $\Phi$ and $\Psi$ satisfy all the regularity assumptions given in Lemma 2.7. In addition, by (2.3) and (2.4) it is easy to verify that $\Phi(0)=\frac{\beta}{\alpha} H\left(a,-\frac{1}{\beta} A\right)+\frac{\sigma}{\gamma} H\left(b, \frac{1}{\sigma} B\right) \geqslant \inf _{X} \Phi \geqslant 0$ and $\Psi(0)=0$ for any $\lambda>0$ and $\mu \geqslant 0$. For any $\lambda \in \Lambda^{\prime}$, we have $\frac{1}{M p}-\lambda A_{0}>0$.

First, we claim that $\delta<+\infty$. To prove this, let $\left\{v_{k}\right\}$ be a sequence of positive numbers such that $v_{k} \rightarrow\left(\left(\operatorname{Mpinf}_{X} \Phi\right)^{\frac{1}{p}}\right)^{+}$as $k \rightarrow \infty$ and

$$
\liminf _{k \rightarrow \infty} \frac{\int_{a}^{b} \sup _{t \in Q\left(v_{k}\right)}\left(F(x, t)+\frac{\mu}{\lambda} G(x, t)\right) d x}{v_{k}^{p}-M p \Phi(0)}=\underset{v \rightarrow\left(\left(M p \inf _{x} \Phi\right)^{\frac{1}{p}}\right)^{+}}{\liminf _{a}} .
$$

Let $r_{k}=\frac{v_{k}^{p}}{M p}$ for each $k \in \mathbb{N}$, then $r_{k} \rightarrow\left(\inf _{X} \Phi\right)^{+}$as $k \rightarrow \infty$. Using the similar method in the proof of Theorem 3.1, we get

$$
\begin{aligned}
\delta=\liminf _{r \rightarrow\left(\inf _{x} \Phi\right)^{+}} \phi(r) & \leqslant \liminf _{k \rightarrow \infty} \phi\left(r_{k}\right) \\
& \leqslant \liminf _{k \rightarrow \infty} \frac{\sup _{v \in \Phi^{-1}\left(-\infty, r_{k}\right)} \Psi(v)}{r_{k}-\Phi(0)} \\
& \leqslant M p \liminf _{k \rightarrow \infty} \frac{\int_{a}^{b} \sup _{t \in Q\left(v_{k}\right)}\left(F(x, t)+\frac{\mu}{\lambda} G(x, t)\right) d x}{v_{k}^{p}-M p \Phi(0)} \\
& =M p \quad \liminf _{v \rightarrow\left(\left(M p \inf _{x} \Phi\right)^{\frac{1}{p}}\right)^{+}} \frac{\int_{a}^{b} \sup _{t \in Q(v)}\left(F(x, t)+\frac{\mu}{\lambda} G(x, t)\right) d x}{v^{p}-M p \Phi(0)} \\
& \leqslant M p\left(A_{0}+\frac{\mu}{\lambda} B_{0}\right)<+\infty .
\end{aligned}
$$

On the other hand, since $\mu \in\left[0, \delta_{g, \lambda}^{\prime}\right)$, we have

$$
\delta \leqslant M p\left(A_{0}+\frac{\mu}{\lambda} B_{0}\right)<M p\left(A_{0}+\frac{\delta_{g, \lambda}^{\prime}}{\lambda} B_{0}\right)=\frac{1}{\lambda}
$$

hence $\Lambda^{\prime} \subset\left(0, \frac{1}{\delta}\right)$. Then for any fixed $\lambda \in \Lambda^{\prime}$, by Lemma 2.7 (c), one of the following alternatives holds:

$\left(c_{1}\right)$ there is a global minimum of $\Phi$ which is a local minimum of $I_{\lambda}$, or

$\left(c_{2}\right)$ there is a sequence $\left\{u_{n}\right\}$ of pairwise distinct critical points (local minima) of $I_{\lambda}$ that converges weakly to a global minimum of $\Phi$.

Now we show that $u_{0}$ is not a local minimum of the functional $I_{\lambda}$, hence the alternative $\left(c_{1}\right)$ does not hold.

Choose $\left\{\mathfrak{u}_{k}\right\} \subset X$ such that $u_{k} \rightarrow u_{0}$ in $X$ as $k \rightarrow \infty$, from (2.3) and (2.4) we have

$$
\begin{aligned}
& \mathrm{I}_{\lambda}\left(\mathfrak{u}_{\mathrm{k}}\right)=\Phi\left(\mathrm{u}_{\mathrm{k}}\right)-\lambda \Psi\left(\mathrm{u}_{\mathrm{k}}\right) \leqslant \frac{1}{\mathrm{mp}}\left(\left\|\mathrm{u}_{\mathrm{k}}^{\prime}\right\|_{\mathrm{L}^{p}}^{\mathrm{p}}+\left(\frac{\alpha}{\beta}\right)^{\mathrm{p}-1}\left|\mathrm{u}_{\mathrm{k}}(\mathrm{a})-\mathrm{A}\right|^{\mathrm{p}}+\left(\frac{\gamma}{\sigma}\right)^{p-1}\left|\mathfrak{u}_{\mathrm{k}}(\mathrm{b})-\mathrm{B}\right|^{p}\right) \\
& -\lambda \int_{a}^{b} F\left(x, u_{k}(x)\right) d x-\mu \int_{a}^{b} G\left(x, u_{k}(x)\right) d x .
\end{aligned}
$$


Since $\lambda \in \Lambda^{\prime}$, (3.9) implies that

$$
\frac{1}{\lambda}<m p \limsup _{\mathfrak{u} \rightarrow u_{0}} \frac{\int_{a}^{b} F(x, u(x)) d x}{\left\|u^{\prime}\right\|_{L^{p}}^{p}+\left(\frac{\alpha}{\beta}\right)^{p-1}|u(a)-A|^{p}+\left(\frac{\gamma}{\sigma}\right)^{p-1}|u(b)-B|^{p}} .
$$

Then there exist $\varepsilon^{\prime}>0$ and $N^{\prime} \in \mathbb{N}$ such that

$$
\frac{1}{\lambda}<\varepsilon^{\prime}<\frac{\int_{a}^{b} F\left(x, u_{k}(x)\right) d x}{\frac{1}{m p}\left(\left\|u^{\prime}\right\|_{L^{p}}^{p}+\left(\frac{\alpha}{\beta}\right)^{p-1}|u(a)-A|^{p}+\left(\frac{\gamma}{\sigma}\right)^{p-1}|u(b)-B|^{p}\right)}
$$

for every $k \geqslant N^{\prime}$, hence $1-\lambda \varepsilon^{\prime}<0$ and

$$
\lambda \varepsilon^{\prime} \frac{1}{m p}\left(\left\|u^{\prime}\right\|_{L^{p}}^{p}+\left(\frac{\alpha}{\beta}\right)^{p-1}|u(a)-A|^{p}+\left(\frac{\gamma}{\sigma}\right)^{p-1}|u(b)-B|^{p}\right)<\lambda \int_{a}^{b} F\left(x, u_{k}(x)\right) d x
$$

for every $k \geqslant N^{\prime}$. In view of $\left(H_{3}^{\prime}\right),(3.10)$, and (3.11), there exists $k>N^{\prime}$ such that

$$
\begin{aligned}
\mathrm{I}_{\lambda}\left(\mathfrak{u}_{\mathrm{k}}\right) & <\left(1-\lambda \varepsilon^{\prime}\right) \frac{1}{\mathrm{mp}}\left(\left\|\mathfrak{u}^{\prime}\right\|_{L^{p}}^{p}+\left(\frac{\alpha}{\beta}\right)^{p-1}|\mathfrak{u}(\mathrm{a})-A|^{p}+\left(\frac{\gamma}{\sigma}\right)^{p-1}|\mathfrak{u}(\mathrm{b})-\mathrm{B}|^{p}\right)-\mu \int_{a}^{b} \mathrm{G}\left(x, u_{k}(x)\right) d x \\
& \leqslant \mathrm{I}_{\lambda}\left(\mathfrak{u}_{0}\right) .
\end{aligned}
$$

Hence $u_{0}$ is not a minimum of $\mathrm{I}_{\lambda}$.

Therefore, by Lemma $2.7\left(c_{2}\right)$, there exists a sequence $\left\{u_{n}\right\}$ of pairwise distinct critical points of $I_{\lambda}$ that converges weakly to $u_{0}$. Since $X$ is compactly embedded into $C([a, b]), u_{n} \rightarrow u_{0}$ uniformly. Finally, taking Remark 2.6 into account completes the proof of the theorem.

\section{Example}

Let $a=0, b=1, \alpha=1, \beta=2, \gamma=2, \sigma=3, h(x, t)=1+x^{2}+\frac{1}{2} \sin t, A=4, B=-5$, then

$$
\mathrm{Q}(v)=\left\{\mathrm{t} \in \mathbb{R}:|\mathrm{t}| \leqslant \frac{1+2^{\frac{1}{q}}}{1-\left(\frac{1}{1+q}\right)^{\frac{1}{q}}} v+4\right\},
$$

and $h:[0,1] \times \mathbb{R} \rightarrow \mathbb{R}$ satisfies the assumptions $(a),(b)$, and $(c)$ with $m=\inf _{(x, t) \in[0,1] \times \mathbb{R}} h(x, t)=\frac{1}{2}, M=$ $\sup _{(x, t) \in[0,1] \times \mathbb{R}} h(x, t)=\frac{5}{2}$. The boundary value problem (1.1) turns to be

$$
\left\{\begin{aligned}
-\left(\phi_{p}\left(u^{\prime}\right)\right)^{\prime}= & \left(\lambda f(x, u(x))+\mu g(x, u(x))+\int_{0}^{u^{\prime}(x)} \frac{\partial}{\partial x}\left(\frac{2 x(1-p)|\tau|^{p-2}}{\left(1+x^{2}+\frac{1}{2} \sin \tau\right)^{2}}\right) d \tau\right) \\
& \times\left(1+x^{2}+\frac{1}{2} \sin u^{\prime}(x)\right), \quad x \in(0,1), \\
u(0)-2 u^{\prime}(0)= & 4, \quad 2 u(1)+3 u^{\prime}(1)=-5 .
\end{aligned}\right.
$$

For the function $f:[0,1] \times \mathbb{R} \rightarrow \mathbb{R}$, we use the assumption in Example 3.1 of [18] directly, that is, we assume that $f(x, t)=\left(x^{2}+1\right) f_{0}(t)$, where

$$
f_{0}(t)= \begin{cases}b_{1}^{3} \sqrt{1-(1-t)^{2}}+1, & t \in\left[0, b_{1}\right] \\ \left(a_{n}-b_{n}^{3}\right) \sqrt{1-\left(a_{n}-1-t\right)^{2}}+1, & t \in \cup_{n=1}^{\infty}\left[a_{n}-2, a_{n}\right] \\ \left(b_{n+1}^{3}-a_{n}\right) \sqrt{1-\left(b_{n+1}-1-t\right)^{2}}+1, & t \in \cup_{n=1}^{\infty}\left[b_{n+1}-2, b_{n+1}\right] \\ 1, & \text { otherwise }\end{cases}
$$


with $b_{1}=2, b_{n+1}=b_{n}^{6}, a_{n}=b_{n}^{4}$ for $n \in \mathbb{N}$. It is obvious that $f$ is an $\mathrm{L}^{1}$-Carathéodary function. According to the argument of Example 3.1 of [18], we know that

$$
\lim _{n \rightarrow \infty} \frac{F_{0}\left(a_{n}\right)}{a_{n}^{2}}=0 \text { and } \lim _{n \rightarrow \infty} \frac{F_{0}\left(b_{n}\right)}{b_{n}^{2}}=+\infty,
$$

where $F_{0}(t)=\int_{0}^{t} f_{0}(s) d s$. Then

$$
A_{\infty}=\liminf _{v \rightarrow+\infty} \frac{\int_{0}^{1} \sup _{t \in Q}(v) F(x, t) d x}{v^{p}}=\liminf _{v \rightarrow+\infty} F_{0}\left(\frac{1+2^{\frac{1}{q}}}{1-\left(\frac{1}{1+q}\right)^{\frac{1}{q}}} v+4\right) \frac{\int_{0}^{1}\left(x^{2}+1\right) d x}{v^{p}}=0
$$

and

$$
\limsup _{t \rightarrow+\infty} \frac{\int_{0}^{1} F(x, t) d x}{\left(\left(\frac{1}{2}\right)^{p-1} 3^{p}+\left(\frac{2}{3}\right)^{p-1} 6^{p}\right) t^{p}}=\frac{\int_{0}^{1}\left(x^{2}+1\right) d x}{\left(\left(\frac{1}{2}\right)^{p-1} 3^{p}+\left(\frac{2}{3}\right)^{p-1} 6^{p}\right)} \limsup _{t \rightarrow+\infty} \frac{F_{0}(t)}{t^{p}}=\infty .
$$

Hence the function $f$ satisfies the assumption $\left(\mathrm{H}_{1}\right)$ in Theorem 3.1. On the other hand, assume that $g(x, t)=\left(1+x^{2}\right)(2+\sin t)$, then

$$
\mathrm{B}_{\infty}=\liminf _{v \rightarrow+\infty} \frac{\int_{0}^{1} \sup _{\mathrm{t} \in \mathrm{Q}(v)} \mathrm{G}(\mathrm{x}, \mathrm{t}) \mathrm{d} x}{v^{\mathrm{p}}}=\liminf _{v \rightarrow+\infty} \frac{\sup _{\mathrm{t} \in \mathrm{Q}(v)}(1+2 \mathrm{t}-\cos \mathrm{t}) \int_{0}^{1}\left(1+x^{2}\right) \mathrm{d} x}{v^{\mathfrak{p}}}=0
$$

and

$$
\liminf _{t \rightarrow+\infty} \int_{0}^{1} G(x, t) d x=\liminf _{t \rightarrow+\infty} \int_{0}^{1}\left(1+x^{2}\right)(1+2 t-\cos t) d x=+\infty .
$$

Hence $g:[0,1] \times \mathbb{R} \rightarrow \mathbb{R}$ satisfies the assumptions $\left(\mathrm{H}_{2}\right)$ and $\left(\mathrm{H}_{3}\right)$ in Theorem 3.1. According to Theorem 3.1, for every $\lambda \in(0,+\infty)$ and every $\mu \in[0,+\infty)$, the boundary value problem (4.1) has an unbounded sequence of classical solutions in $\mathrm{C}([0,1])$.

\section{Acknowledgment}

The authors are supported financially by the National Natural Science Foundation of China (11371221, 11571296).

\section{References}

[1] G. A. Afrouzi, G. Caristi, D. Barilla, S. Moradi, A variational approach to perturbed three-point boundary value problems of Kirchhoff-type, Complex Var. Elliptic Equ., 62 (2017), 397-412. 1

[2] D. Averna, G. Bonanno, Three solutions for a quasilinear two-point boundary value problem involving the one-dimensional p-Laplacian, Proc. Edinb. Math. Soc., 47 (2004), 257-270. 1

[3] G. Barletta, A. Chinnì, D. O'Regan, Existence results for a Neumann problem involving the $\mathrm{p}(\mathrm{x})$-Laplacian with discontinuous nonlinearities, Nonlinear Anal. Real World Appl., 27 (2016), 312-325.

[4] G. Bonanno, Multiple critical points theorems without the Palais-Smale condition, J. Math. Anal. Appl., 299 (2004), 600-614. 1

[5] G. Bonanno, P. Candito, Non-differentiable functionals and applications to elliptic problems with discontinuous nonlinearities, J. Differential Equations, 244 (2008), 3031-3059. 1

[6] G. Bonanno, B. Di Bella, J. Henderson, Infinitely many solutions for a boundary value problem with impulsive effects, Bound. Value Probl., 2013 (2013), 14 pages. 1

[7] G. Bonanno, N. Giovannelli, An eigenvalue Dirichlet problem involving the $\mathrm{p}$-Laplacian with discontinuous nonlinearities, J. Math. Anal. Appl., 308 (2005), 596-604. 1

[8] G. Bonanno, P. Jebelean, C. Şerban, Superlinear discrete problems, Appl. Math. Lett., 52 (2016), 162-168. 1

[9] G. Bonanno, G. Molica Bisci, V. Rădulescu, Multiple solutions of generalized Yamabe equations on Riemannian manifolds and applications to Emden-Fowler problems, Nonlinear Anal. Real World Appl., 12 (2011), 2656-2665. 1

[10] H. Brezis, Functional Analysis, Sobolev Spaces and Partial Differential Equations, Springer, New York, (2011). 2,2 
[11] H. Chen, J. Sun, An application of variational method to second-order impulsive differential equation on the half-line, Appl. Math. Comput., 217 (2010), 1863-1869. 1

[12] J.-F. Chu, F. Gharehgazlouei, S. Heidarkhani, A. Solimaninia, Three nontrivial solutions for Kirchhoff-type variationalhemivariational inequalities, Results Math., 68 (2015), 71-91. 1

[13] G. D'Aguì, J. Mawhin, A. Sciammetta, Positive solutions for a discrete two point nonlinear boundary value problem with p-Laplacian, J. Math. Anal. Appl., 447 (2017), 383-397. 1

[14] G. D'Aguì, A. Sciammetta, Infinitely many solutions to elliptic problems with variable exponent and nonhomogeneous Neumann conditions, Nonlinear Anal., 75 (2012), 5612-5619. 1

[15] G. Dai, Three solutions for a nonlocal dirichlet boundary value problem involving the $\mathrm{p}(\mathrm{x})$-Laplacian, Appl. Anal., 92 (2013), 191-210. 1

[16] F. Faraci, G. Smyrlis, Three solutions for a class of higher dimensional singular problems, NoDEA Nonlinear Differential Equations Appl., 2016 (2016), 14 pages. 1

[17] J. R. Graef, S. Heidarkhani, L.-J. Kong, A critical points approach for the existence of multiple solutions of a Dirichlet quasilinear system, J. Math. Anal. Appl., 388 (2012), 1268-1278. 1

[18] J. R. Graef, S. Heidarkhani, L.-J. Kong, Infinitely many solutions for systems of Sturm-Liouville boundary value problems, Results Math., 66 (2014), 327-341. 1, 3.3, 4

[19] S. Heidarkhani, G. A. Afrouzi, M. Ferrara, S. Moradi, Variational approaches to impulsive elastic beam equations of Kirch hoff type, Complex Var. Elliptic Equ., 61 (2016), 931-968. 1

[20] S. Heidarkhani, M. Ferrara, S. Khademloo, Nontrivial solutions for one-dimensional fourth-order Kirchhoff-type equations, Mediterr. J. Math., 13 (2016), 217-236. 1

[21] J. Liu, Z. Zhao, Multiple solutions for impulsive problems with non-autonomous perturbations, Appl. Math. Lett., 64 (2017), 143-149. 1

[22] S. A. Marano, D. Motreanu, On a three critical points theorem for non-differentiable functions and applications to nonlinear boundary value problems, Nonlinear Anal., 48 (2002), 37-52. 1

[23] B. Ricceri, A general variational principle and some of its applications, J. Comput. Appl. Math., 113 (2000), 401-410. 1, 2.7

[24] B. Ricceri, On a three critical points theorem, Arch. Math. (Basel), 75 (2000), 220-226. 1

[25] B. Ricceri, A three critical points theorem revisited, Nonlinear Anal., 70 (2009), 3084-3089. 1

[26] B. Ricceri, On an elliptic Kirchhoff-type problem depending on two parameters, J. Global Optim., 46 (2010), 543-549. 1

[27] R. Rodríguez-López, S. Tersian, Multiple solutions to boundary value problem for impulsive fractional differential equations, Fract. Calc. Appl. Anal., 17 (2014), 1016-1038. 1

[28] Y. Tian, W. Ge, Multiple solutions for a second-order Sturm-Liouville boundary value problem, Taiwanese J. Math., 11 (2007), 975-988. 1, 2

[29] Y. Tian, J. R. Graef, L. Kong, M. Wang, Three solutions for second-order impulsive differential inclusions with SturmLiouville boundary conditions via nonsmooth critical point theory, Topol. Methods Nonlinear Anal., 47 (2016), 1-17. 1

[30] L. Yang, H. Chen, X.-X. Yang, The multiplicity of solutions for fourth-order equations generated from a boundary condition, Appl. Math. Lett., 24 (2011), 1599-1603. 1

[31] E. Zeidler, Nonlinear Functional Analysis and Its Applications, Springer, New York, (1988). 2

[32] X. Zhang, L. Liu, Y. Wu, B. Wiwatanapataphee, Nontrivial solutions for a fractional advection dispersion equation in anomalous diffusion, Appl. Math. Lett., 66 (2017), 1-8. 1 